\title{
The Connection Matrix analysis of a Prey-Predator system with a Strong Allee effect and multiple interior equilibria
}

\section{Asim Sikder ( $\sim$ asim_sikder@rediffmail.com )} Jadavpur University https://orcid.org/0000-0002-7065-6705

\section{Research Article}

Keywords: Gause-type prey-predaror system, allee effect for the prey, Multiple interior equilibria, saddle to saddle connections, the Conley connection matrix, permanence

Posted Date: November 30th, 2021

DOI: https://doi.org/10.21203/rs.3.rs-851623/v1

License: (a) (i) This work is licensed under a Creative Commons Attribution 4.0 International License.

Read Full License 


\title{
The Connection Matrix analysis of a Prey-Predator system with a Strong Allee effect and multiple interior equilibria
}

\author{
Asim Sikder * \\ Department of Mathematics, \\ Jadavpur University, \\ kolkata-700032 \\ INDIA
}

\begin{abstract}
We consider a Gause-type prey-predator system incorporating a strong allee effect for the prey population. For the existence of multiple interior equilibria we consider Holling-type predator functional response and the density dependent death rate for the predator. With the help of the Conley connection matrix theory we study the dynamics of the system in presence of one, two and three interior equilibria. It is found that (i) the saddle-saddle connections exist in presence of single and multiple interior equilibria connecting interior flows to the boundary and (ii) the system admits a set of degree-2 (i.e, a 2-discs of) connecting orbits from interior equlibrium to the origin. Thus permanence or robust permanence of the system is not possible.
\end{abstract}

keyword: Gause-type prey-predaror system, allee effect for the prey, Multiple interior equilibria, saddle to saddle connections, the Conley connection matrix, permanence.

Running title : Prey-Predator system With Allee effect

*E-mail address: asim _sikder@rediffmail.com 


\section{Introduction}

We consider the following predator prey system with strong allee effect in the prey growth, Holling type II functional response and density dependent death rate of predators.

$$
\begin{aligned}
& x^{\prime}=x g(x)-y p(x) \\
& y^{\prime}=y[c p(x)-d-h y],
\end{aligned}
$$

where $x(t)$ and $y(t)$ are the prey and predator population density at time t, respectively with $x(0), y(0) \geq 0 . g(x)$ is the per capita growth rate of the prey in absence of the predator. $p(x)$ is the Holling type II functional response for the predaror. $d+h y$ is the density dependent death rate of the predator. $d, h$ are positive constants.

The biological/ecological explanations and/or other related ideas may be found in details in $[4,12,16,17]$. Conway and Smoller [2] studied the global dynamics of the prey- predator equations with allee effect for the prey using the stable and unstable manifolds of the axial equilibria, that is, by the separatrices and established the intersection of these manifolds for some unique value of a parameter using the properties of the prey and the predator isoclines. van Voorn et. al, [16] analysed two predator-prey models with strong allee effect with bifurcation analysis and showed the existence of saddle-saddle connections in these models and the systems collapse after the invasion of the predators. Wang et. al, [17] considered a class of general predator-prey models with a strong allee effect in prey population and showed the existence of point to point heteroclic orbit loop with the general properties of the separatries, i.e, the stable and unstable manifolds of the two prey equilibria and proved the existence/uniqueness and the nonexistence of limit cycles for appropriate range of parameters. With the model (1) in explicit form (see the discussion), Sen and Banerjee [12] studied the change in the number of interior equilibria and their stability properties by bifurcation analysis.

In this paper we first prove the existence of multiple interior equilibria of our model(1) and then establish the saddle-saddle connections in presence of a single interior equilirium and also in presence of multiple interior equilibria from purely algebraic way using the Conley connection matrix theory, see [3, $7,8,9]$. We also establish a set of degree-2 connecting orbits, i, e, a set of 2-discs of connecting orbits from the interior equilibrium to the origin. To do so we need only the existence of the equilibria and their local stability dimensions and show the movement of our system interior flows using the Conley connection matrix without any phase plane analysis. Reineck [9] studied the ecological models using the connection matrix analysis, mainly in two dimension. Also see the papers of Mischaikow [7, 8]. By the connection matrix analysis in four species systems the present author found interesting results in $[13,14]$ establishing the structure of the connecting orbits.

In section 2, we state the basic assumptions regarding our system, prove the existence of multiple interior equilibria and other results. In the final section we add some remarks and discussion regarding the results of the system. 


\section{Assumptions and Results:}

(H1) $g(x) \in C^{1}([0,+\infty)), g(b)=g(K)=0$ with $0<b<K, g(x)$ is positive for $b<x<K$ and negative otherwise. $g^{\prime}(x)>0$ on $[b, m)$, and $g^{\prime}(x)<0$ on $(m, K] . g^{\prime \prime}(x)<0$.

$K$ is the carrying capacity for the prey. In the absence of the predator the prey $x$ has a strong allee effect.

(H2) $p(x) \in C^{1}([0,+\infty)), p(0)=0, p^{\prime}(x)>0$ for $x \geq 0$. There exists $\bar{x}$ such that $c p(\bar{x})=d$.

By the above assumption the prey isocline $x^{\prime}=0$ lies in $\mathbf{R}_{+}^{2}$ for $x \in[b, K]$ and a continuous smooth curve joining the points $(b, 0)$ and $(K, 0)$ having a local maximum at $x=m$. The predator isocline $y^{\prime}=0$ (Holling type II) intersects the positive $x$ axis at $(\bar{x}, 0)$ and then increases monotonically. The $x$ axis and the $y$ axis of system (1) are invariant and thus the interior of $R_{+}^{2}$ is also invariant.

Lemma 0.1 Suppose (H1) - (H2) holds. Then the solutions of system (1) are uniformly bounded.

Proof :See $[12,13]$

The system (1) has the boundary equilibria: a) The origin $E_{0}$, which always exists and $\mathbf{b})$ two equilibria on the prey-axis, $E_{1}(b, 1)$ and $E_{2}(K, 0)$. They always exist by $(H)$. Next we show that the system (1) has one or more equilibria in the interior of the positive quadrant using the Index theorem for ecological equations(see [5]) An equilibrium for an ecological equation $x^{\prime}=x f(x)$ is saturated (nonsaturated) if its external eigenvalue is nonnegative (positive). A point $x \in R_{+}^{2}$ is called regular if $\operatorname{det} D_{x} f \neq 0$. From the Jacobian matrix of (1), it is trivial that i) $E_{0}$ is always locally asymptotically stable, ii) $E_{1}$ is unstable (i,e nonsaturated ) along the predator direction if $0<\bar{x}<b$ and stable, i.e, saturated along the predator direction if $b<\bar{x}<K$, and iii) $E_{2}$ is unstable along the $y$ direction if $0<\bar{x}<K$.

Lemma 0.2 Suppose that (H1) and (H2) holds.

(i) If $0<\bar{x}<b$, there exists no interior equilibrium or two interior equilibria, $E(1)$ and $E(2)$, (ii) If $b<\bar{x}<K$, there exists one or three interior equilibria $E(i)$ for $i=1,2,3$

Proof (i) In this case the boundary equilibria $E_{0}$ is saturated, and $E_{1}, E_{2}$ are nonsaturated. $\operatorname{Ind}\left(E_{0}\right)=+1$. $\left[\operatorname{Ind}\left(E_{0}\right)\right.$ means the index of $E_{0}$ and the index of any equilibrium is $(-1)^{n}$, where $n$ is the dimension of the stable manifold of the equilibrium.] Since our system is $2 \mathrm{D}$ and its solutions are uniformly bounded, the sum of indices of all its saturated regular fixed points is $(-1)^{2}=+1$ by the Index theorem for ecological equations (see [5]). Thus there exists no interior equilibrium or two interior equilibria $E(i)$, with $\operatorname{Ind}(E(i)=+1$ or -1 for $i=1,2$. There are no other possible interior equilibrium due to $(\mathrm{H})$.

(ii) $E_{0}, E_{1}$ are saturated and $E_{2}$ is nonsaturated. $\operatorname{Ind}\left(E_{0}\right)=+1$ and $\operatorname{Ind}\left(E_{1}\right)$ $=-1$. To make the sum of indices of all saturated equilibria is $(+1)$, there 
must exist at most one (with index $(+1)$ ) or three interior fixed points. Out of three, at least one is a saddle point with index $(-1)$, one is either a sink or source, or center with index $(+1)$ and the rest one must be either a sink or source, or center with index $(+1)$.

In our analysis we will not consider that the system (1) has a fixed point which is center with index $(+1)$. For our system we consider

$$
N=\{(x, y):-a \leq x, y \leq M\},
$$

(where $a>0$ and $M$ is sufficiently large) is an isolated invariant neighbouhood for some set $S$ containing in the positive quadrant. The equilibria $E_{0}, E_{1}, E_{2}$ and $E(i)$, where $i=1$ or $1,2,3$ are the Morse sets. In the positive quadrant there may or may not exist periodic solutions. Thus we define $(\pi)$ to be the minimal isolated invariant set in the interior of the positive quadrant containing all periodic orbits of $S$ (if exist). (See[8].) Using $\mathrm{Z}_{2}$ coefficients the homologies of the conley indices of these equilibria are given by

$$
\begin{gathered}
C H_{k}\left(E_{1}\right)=C H_{k}\left(E_{2}\right)=\left\{\begin{array}{cc}
\mathbf{Z}_{2} & \text { if } k=1 \\
0 & \text { otherwise }
\end{array}\right. \\
C H_{k}\left(E_{0}\right)=\left\{\begin{array}{cc}
\mathbf{Z}_{2} & \text { if } k=0 \\
0 & \text { otherwise }
\end{array}\right.
\end{gathered}
$$

and

$$
\text { for } i=1,2,3, C H_{k}(E(i))=\left\{\begin{array}{cc}
\mathbf{Z}_{2} & \text { if } k=2,1, \text { or } 0 \\
0 & \text { otherwise }
\end{array}\right.
$$

Theorem 0.1 Suppose That (H1) and (H2) holds.

(a)Let $b<\bar{x}<K$ and the isoclines $x^{\prime}=y^{\prime}=0$ intersect at a unique point $E$. Then one of the following hold

(i) There exist a heteroclinc orbit $E_{2} \rightarrow E_{1}$.

(ii) There exists a set of degree -2 connecting orbits from $E$ to $E_{0}$.

(b)Let $b<\bar{x}<K$ and the isoclines $x^{\prime}=y^{\prime}=0$ intersect at three points $E(i), i=1,2,3$. Then there exists a 2-disc of connections from $E(1)$ to $E_{0}$.

(c) Let $0<\bar{x}<b$ and the prey, predator isoclines intersect at two pionts $E(1)$ and $E(2)$. Then there exists a heteroclinc orbit either $E_{1} \rightarrow E(1)$. or $E_{1} \rightarrow E_{2}$.

Proof: (i) Consider the equilibrium $E$ lies on the decreasing portion or on the increasing portion of the prey isocline, i.e, it may be sink or source and around it there are periodic orbits. Also consider the homology index $C H_{*}(\pi E)=$ $\left(\mathbf{Z}_{2}, 0,0, \ldots\right)$, if $(\pi E)$ is an attracting interval. Then the Morse sets $E_{0}, E_{1}, E_{2}$, and $(\pi E)$ form a Morse decomposition and the connection matrix of these sets is of the form 


$$
\begin{gathered}
E_{0}(\pi E) \\
A=\begin{array}{c}
E_{1} \\
E_{0} \\
(\pi E) \\
E_{1} \\
E_{2}
\end{array}\left[\begin{array}{llll}
0 & 0 & 1 & * \\
0 & 0 & 0 & 1 \\
0 & 0 & 0 & 0 \\
0 & 0 & 0 & 0
\end{array}\right]
\end{gathered}
$$

The entry $*$ is not known. The connections $E_{1} \rightarrow E_{0}$ and $E_{2} \rightarrow(\pi E)$ come from linear analysis. The zero entries of $A$ are due to degree considerations. The entries of $A$ are maps of degree -1 . Because of $A^{2}=0$, the value of $*$ is equal to zero or one, i.e, we can have two connection matrices $A_{0}$ with $*=0$ and $A_{1}$ with $*=1$. With these two connection matrices the connection matrix for the product space is of the form

$$
\left[\begin{array}{cc}
A_{0} & T \\
0 & A_{1}
\end{array}\right]
$$

where $T$ is the transition matrix and by Lemma 2.19 [9] and the square zero condition implies that $T$ will be of the following form

$$
\left[\begin{array}{llll}
1 & 0 & 0 & 0 \\
0 & 1 & 0 & 0 \\
0 & 0 & 1 & 1 \\
0 & 0 & 0 & 1
\end{array}\right]
$$

This gives us the connection $E_{2} \rightarrow E_{1}$, i.e, as the system is deformed from one flow (which gives $A_{0}$ connection matrix) to another flow (which gives $A_{1}$ connection matrix) we get the saddle-saddle connection $E_{2} \rightarrow E_{1}$. Moreover, Theorem 2.20 [9] shows that there is a sequence of connections at different parameter values starting at $E_{2}$ and ending at $E_{1}$. We note that the connection $E_{1} \rightarrow E_{2}$ can not be obtained from the above connection matrix for the product space. This is because that connection is not transversal. It comes from the linear analysis and it lies on the prey axis. It is to be noted here that there are other possible transition matrices for the product space but we are not considering those, because we are only interested for the matrices that involve only a single saddle-saddle connection via which the interior flows can converge to the boundary

(ii) Consider $E$ lies on the increasing portion of the prey isocline and it is source and there are no periodic orbits around it. The remaining equilibria are: $E_{0}$ sink, $E_{1}$ saddle, $E_{2}$ saddle. The connection matrix is

$$
B=\begin{array}{cccc}
E_{0} & E_{1} & E_{2} & E \\
E_{0} \\
E_{1} \\
E_{2} \\
E
\end{array}\left[\begin{array}{llll}
0 & 1 & 1 & \star \\
0 & 0 & 0 & 1 \\
0 & 0 & 0 & 1 \\
0 & 0 & 0 & 0
\end{array}\right]
$$


Now $B^{2}=0$ implies that $\star=1+1=0$. Since $\Delta\left(E_{1}, E_{0}\right) \circ \Delta\left(E, E_{1}\right) \neq 0$ and $\Delta\left(E_{2}, E_{0}\right) \circ \Delta\left(E, E_{2}\right) \neq 0$, Theorem 3.2 of Mischaikow and Reineck [7] gives us a set of degree -2 connecting orbits from $E$ to the origin $E_{0}$, that is, the unstable manifold of $E$ intersects the stable manifold of $E_{0}$ in a two-dimentional set. The boundary of this $2 \mathrm{D}$ set consists of connections $E \rightarrow E_{1}$ and $E \rightarrow E_{2}$. (The connections $E_{1} \rightarrow E_{0}, E \rightarrow E_{1}$, come from the linear analysis and the connections $E \rightarrow E_{1}$ and $E \rightarrow E_{2}$. are from Theorem 3.2 [7]).

Now consider there exists periodic orbits around $E$, and the homology indices of $(\pi E)$ is $\left(0,0, \mathbf{Z}_{\mathbf{2}}, 0,0, \ldots\right)$ then we can replace the Morse set $E$ by the Morse set $(\pi E)$ and we will get the same result.

(b) In this case consider $E(1)$ source, $E(2)$ saddle and $E(3)$ sink. Also consider there are periodic about $E(3)$ and we denote the Morse set $(\pi E(3))$ by $M$. The homology index of $(\pi E(3))$ or $M$ is $\left(\mathbf{Z}_{\mathbf{2}}, 0,0, \ldots.\right)$. Then the connection matrix is

$$
\begin{gathered}
E_{0} \\
D= \\
E_{0} \\
M \\
E_{1} \\
E_{2} \\
E(2) \\
E(1)
\end{gathered}\left[\begin{array}{rrrrrr}
0 & 0 & 1 & * & * & * \\
0 & 0 & 0 & 1 & 1 & * \\
0 & 0 & 0 & 0 & 0 & 1 \\
0 & 0 & 0 & 0 & 0 & * \\
0 & 0 & 0 & 0 & 0 & 0 \\
0 & 0 & 0 & 0 & 0 & 0
\end{array}\right]
$$

Using the condition that $D^{2}=0$ and $\operatorname{rank} D=3$ we get the following connection matrices

$$
\begin{aligned}
& \begin{array}{llllll}
E_{0} & M & E_{1} & E_{2} & E(2) & E(1)
\end{array} \\
& D_{1}=\begin{array}{c}
E_{0} \\
M \\
E_{1} \\
E_{2} \\
E(2) \\
E(1)
\end{array}\left[\begin{array}{llllll}
0 & 0 & 0 & 1 & 0 & 0 \\
0 & 0 & 0 & 1 & 1 & 0 \\
0 & 0 & 0 & 0 & 0 & 1 \\
0 & 0 & 0 & 0 & 0 & 0 \\
0 & 0 & 0 & 0 & 0 & 0 \\
0 & 0 & 0 & 0 & 0 & 0
\end{array}\right] \\
& \begin{array}{llllll}
E_{0} & M & E_{1} & E_{2} & E(2) & E(1)
\end{array} \\
& D_{2}=\begin{array}{c}
E_{0} \\
M \\
E_{1} \\
E_{2} \\
E(2) \\
E(1)
\end{array}\left[\begin{array}{llllll}
0 & 0 & 0 & 0 & 1 & 0 \\
0 & 0 & 0 & 1 & 1 & 0 \\
0 & 0 & 0 & 0 & 0 & 1 \\
0 & 0 & 0 & 0 & 0 & 0 \\
0 & 0 & 0 & 0 & 0 & 0 \\
0 & 0 & 0 & 0 & 0 & 0
\end{array}\right]
\end{aligned}
$$




$$
\begin{aligned}
& \begin{array}{llllll}
E_{0} & M & E_{1} & E_{2} & E(2) & E(1)
\end{array} \\
& D_{3}=\begin{array}{c}
E_{0} \\
M \\
E_{1} \\
E_{2} \\
E(2) \\
E(1)
\end{array}\left[\begin{array}{llllll}
0 & 0 & 0 & 1 & 1 & 0 \\
0 & 0 & 0 & 0 & 1 & 0 \\
0 & 0 & 0 & 0 & 0 & 1 \\
0 & 0 & 0 & 0 & 0 & 0 \\
0 & 0 & 0 & 0 & 0 & 0 \\
0 & 0 & 0 & 0 & 0 & 0
\end{array}\right]
\end{aligned}
$$

From $D_{1}$ consider the subset $\left\{E_{0}, E_{1}, E(1)\right\}$. Since there is always an connection from $E_{1} \rightarrow E_{0}$ (see $D$ ), the zero entry in $D_{1}$ from $E_{1}$ to $E_{0}$ says that there is a double connections $E_{1} \rightarrow E_{0}$. Now we can consider a compact set $N$ which is a neighborhood of $E_{0}, E_{1}, E(1)$, the connection $E(1) \rightarrow E_{1}$ and one of the connections $E_{1} \rightarrow E_{0}$. By Theorem 3.1 of Mischaikow and Reinneck [7], $N$ cannot be an isolating neighborhood (since the connection from $E_{1}$ to $E_{0}$ is a single one) and there must be a boundary point of $N$ which lies on the connection $E(1) \rightarrow E(2)$. We will get the same conclusion if we expand $N$ as long as the boundary maps give the same connection matrix. This occurs in two ways; either the second connection $E_{1} \rightarrow E_{0}$ is in $N$ or $N$ contains one connection $E_{1} \rightarrow E_{0}$, one equilibrium $E(2)$ with the connections $E(2) \rightarrow E_{0}$ and $E(1) \rightarrow E(2)$. Then by Theorem 3.2 of [7] there is a 2-disc of connections $E(1) \rightarrow E_{0}$ and the boundary of the disc contain the connections $E_{1} \rightarrow E_{0}$ and $E(2) \rightarrow E_{0}$. (See figure 3.) Similarly if we consider the subset $\left\{E_{0}, E_{1}, E(1)\right\}$ from $D_{2}$ and from $D_{3}$, we get in similar ways a disc of connections from $E(1)$ to $E_{0}$.

(c) In this case cosider $E(1)$ saddle and $E(2)$ sink. Consider there are periodic solutions around the equilibrium $E(2)$. We also denote the Morse set $(\pi E(2))$ by $M$. The Morse decomposition consists of equilibria $E_{0}, E_{1}, E_{2}, E(1)$ and $M$ and the connection matrix is of the form

$$
\begin{aligned}
& E_{0} \quad M \quad E_{1} \quad E_{2} \quad E(1) \\
& C=\begin{array}{c}
E_{0} \\
M \\
E_{1} \\
E_{2} \\
E(1)
\end{array}\left[\begin{array}{lllll}
0 & 0 & 1 & * & * \\
0 & 0 & * & 1 & 1 \\
0 & 0 & 0 & 0 & 0 \\
0 & 0 & 0 & 0 & 0 \\
0 & 0 & 0 & 0 & 0
\end{array}\right]
\end{aligned}
$$

The connections from $E_{1} \rightarrow E_{0}, E_{2} \rightarrow M$ and $E(1) \rightarrow M$ are from linear analysis. Using the condition that $B^{2}=0$ and $\operatorname{rank} B=3$, we obtain 4 possible connection matrices listed by $B_{i}$ for $i=1,2,3,4$.

$$
C_{1}=\left[\begin{array}{lllll}
0 & 0 & 1 & 0 & 0 \\
0 & 0 & 1 & 1 & 1 \\
0 & 0 & 0 & 0 & 0 \\
0 & 0 & 0 & 0 & 0
\end{array}\right], C_{2}=\left[\begin{array}{lllll}
0 & 0 & 1 & 1 & 0 \\
0 & 0 & 1 & 1 & 1 \\
0 & 0 & 0 & 0 & 0 \\
0 & 0 & 0 & 0 & 0
\end{array}\right], C_{3}=\left[\begin{array}{lllll}
0 & 0 & 1 & 0 & 1 \\
0 & 0 & 1 & 1 & 1 \\
0 & 0 & 0 & 0 & 0 \\
0 & 0 & 0 & 0 & 0
\end{array}\right],
$$


and

$$
C_{4}=\left[\begin{array}{lllll}
0 & 0 & 1 & 0 & 0 \\
0 & 0 & 0 & 1 & 1 \\
0 & 0 & 0 & 0 & 0 \\
0 & 0 & 0 & 0 & 0
\end{array}\right] .
$$

The connection matrix $C_{2}$ is not possible, since the connection $E_{2} \rightarrow E_{0}$. needs to cross the unstable manifold of $E_{1}$ which violates the uniqueness property. The unstable manifold of $E_{1}$ lies above the prey isocline $x^{\prime}=0$ (see [3, 17]).

Similarly, the connection matrix $C_{3}$ is not possible as then $E(1) \rightarrow E_{0}$. would cross the unstable manifold of $E_{1}$. Otherwise, whenever both $C_{2}$ and $C_{3}$ are possible then the unstable manifolds of $E_{1}$ and $E_{2}$ or $E(1)$ move to $E_{0}$. However we consider the matrices $C_{1}, C_{4}$ and form the connection matrix for the product flow

$$
\left[\begin{array}{cc}
C_{1} & T_{i} \\
0 & C_{4}
\end{array}\right]
$$

where $T_{i}$ is the two possible transition matrices by Lemma 2.19 [9] and the square matrix zero condition says that $T_{i}$ will be of the following form

$$
T_{1}=\left[\begin{array}{lllll}
1 & 0 & 0 & 0 & 0 \\
0 & 1 & 0 & 0 & 0 \\
0 & 0 & 1 & 0 & 0 \\
0 & 0 & 0 & 1 & 0 \\
0 & 0 & 1 & 0 & 1
\end{array}\right], \quad \text { and } \quad T_{2}=\left[\begin{array}{lllll}
1 & 0 & 0 & 0 & 0 \\
0 & 1 & 0 & 0 & 0 \\
0 & 0 & 1 & 0 & 0 \\
0 & 0 & 1 & 1 & 0 \\
0 & 0 & 0 & 0 & 1
\end{array}\right]
$$

From $T_{1}$ we get the connection $E_{1} \rightarrow E(1)$, as the system is deformed from one flow (which gives $C_{1}$ connection matrix) to another flow (which gives $C_{4}$ connection matrix). (See figure 1). From $T_{2}$ we get the saddle-saddle connection $E_{1} \rightarrow E_{2}$. (not along the prey axis, because the unstable manifold of $E_{1}$ lies above the prey isocline.) (See figure 2). It is to be noted here that there are other possible transition matrices for the product space but we are not considering those, because we are only interested for the matrices that involve only a single saddle-saddle connection via which the interior flows can converge to the boundary.

If we form the connection matrices with $C_{1}$ and $C_{2}$, and with $C_{1}$ and $C_{3}$ for the product flow then the condition that the square of the matrix is zero fails, that is we will not get any single saddle-saddle connection.

\section{Discussion and Remarks}

1. The exclusion of periodic orbits around a unique equilibrium can be obtained by Theorem 1 of Liu Y [6]. We consider the model studied in $[12]$

$$
\begin{aligned}
x^{\prime} & =x[(1-x)(x / L-1)-\beta /(x+\alpha)] \\
y^{\prime} & =y[\beta x-\gamma-\delta y],
\end{aligned}
$$

By Lemma 0.2, the system (2) has no interior equilibrium or two interior equilibria if $\alpha \gamma /(\beta-\gamma)<L$, and contains one or three interior equilibrium 
if $L<\alpha \gamma /(\beta-\gamma)<1$. The system admits no periodic solutions around the unique equilibrium $E^{\star}$ if

$$
\text { i) } \alpha>L+1
$$

and

$$
\text { ii) } F^{\prime}\left(E^{\star}\right) \leq 0
$$

where

$$
F(x)=1 / \beta(1-x)(x / L-1)(x+\alpha) .
$$

The condition (ii) is not difficult to check, whenever $E^{\star}$ lies on the decreasing part of the prey isocline.

2. Presence of multiple interior equilibria (two or three) changes the dynamics of the system from that of dynamics the system admits with a single interior equilibrium (see Figs. 1-3). For an example, when the system admits three interior equilbria we get a 2-disc of connecting orbits which is different (containing a different connection orbit along its boundary) in case with a single fixed point and due to its presence the saddle-saddle connection $E_{2} \rightarrow E_{1}$ does not occur in any case.

3. The existence of $E_{2} \rightarrow E_{1}$ comes from the connection matrix and that matrix is due to the Morse decomposition including the Morse set $E$ or $(\pi E)$ and $E$ is sink or $(\pi E)$ is the attracting interval, not all orbits inside the heteroclinic orbit $E_{2} \rightarrow E_{1}$ can converge to this heteroclinc orbit. In particular some solutions near $E$ or $(\pi E)$ may converge to $E$ or $(\pi E)$. This is in contrast with the result: Theorem $4.4(2)$ in [17]

4. For the connection matrix $A_{1}$ in Theorem 0.1 (a) there exist the connections $E_{2} \rightarrow E_{0}$ and $E_{2} \rightarrow E$. In the plane there is obviousely no such $E_{2} \rightarrow E_{0}$ connection but it is forced by Morse decomposition, i. e., this comes from the connection matrix which is not a planar phenomenon. Moreover, the connection $E_{2} \rightarrow E_{1}$ reveals the fact that the unstable manifold of $E_{2}$ moves above the stable manifold of $E_{1}$ ( also above the prey isocline) to get access to move to $E_{0}$, until they intersect at some parameter value of the system.

5. The connection $E_{2} \rightarrow E_{1}$ is obtained in almost all studies $[3,16,17]$ but with different approaches. We obtain the same connection by the Conley connection matrix analysis in presence of one or two interior fixed points. We also get a set of 2-discs of connecting orbits from interior fixed point to the origin. However, in any case the interior flows of such $2 \mathrm{D}$ systems moves to the extinction state, i.e, to the boundary. Thus the permanence [1] or robust permanence [11] of the system is not possible. That is, the biological significance of our results is that the allee effect for the prey does not promote the system coexistence in any case. Moreover, the structure of the attractor of this impermanent $2 \mathrm{D}$ system is either a 2disced shape set or a shape of a heteroclinic loop intersecting the interior and the boundary. [10]. 


\section{References}

[1] Butler, G. J., Freedman, H. I., Waltman, P. : Uniformly persistence systems, Proc. Am. Math. Soc. 96, 425-430 (1980).

[2] Conway, E. D., Smoller, J. A.: Global analysis of a of a system of predatorprey equations, SIAM Journal on Applied Mathematics 46, 630-642 (1986)

[3] Franzosa, R.: The connection matrix theory for Morse decomposition, Trans.Am.Soc. 311, 561-592 (1989)

[4] Gonzalez-Olivares, E. Rojas-Palmer A.:Allee Effect in Gause-tpe PredatorPrey Models:Existence of multiple Attractors, Limit cycles and Separatrix Curves. A brief review, Math. Model. Nat. Phenom,6, 143-164 (2013)

[5] Hofbauer, J.,Sigmund, K.; The Theory of Evolution and Dynamical System, Cambridge University Press, Cambridge 1988

[6] Liu, Y., Geometri criteria for the Nonexistence of Cycles in Gause-type Predator-Prey Systems, Pro. Am. Math. Soc. 133, 3619-3626 (2005)

[7] Mischaikow, K., Reineck, J. F., A product theorem for connection matrices and the structure of connecting orbits, JNA-TMA, 23,1293-1314 (1994)

[8] Mischaikow, K., Wolkowicz, G., A Predator- Prey System involving Group Defence: a Connection matrix approach, JNA-TMA, 14, 955-969, (1990)

[9] Reineck, J., F., A connection matrix analysis of ecological models, JNATMA,23 1293-1314 (1991)

[10] Sanjurjo, J. M. R., On the fine structure of the global attractor of a uniformly persistence, J. Differ. Eq.,252 4886-4897 (2012)

[11] Schreiber, S.,Criteria for $C^{r}$ robast permanence, J. Differ. EQ. $162400-$ 426 (2002)

[12] Sen, M; Banerjee M., Rich dynamics in a prey-predator model, Int. J. Bifur. Chaos, with Allee effect and density dependent death rate of predators,

[13] Sikder, A., Roy, A. B.: Persistence of a Generalised Gause-type peypredator pair linked by competition, Math. Biosci.,122 1-23 (1994)

[14] Sikder, A., The connection matrix and heteroclinic cycles in two preypredator pairs with multiple equilibria, Nonlinear analysis: RWA, 3 583$595(2002)$

[15] Sikder, A., A lotka-Volterra competition model and its global convergence ti a definite axial equilibrium, J. Math.,Biol, 44, 297-308 (2004)

[16] van Voorn, G., A., Hererik, L.,Boer, M.P, Kooi, B. W., Heteroclinc orbits indicate overexploitation in prey-predator systems with a strong allee effect, Math. Biosci. 209,451-469 (2007) 
[17] Wang, J.,Shi, J., Wei, J.,, Ptrdator-prey system with Strong allee effect in prey, J. Math. Biol.,62, 291-331 (2011) 
Figure legend:

Fig. 1 This figure shows the existence of the heteroclinic orbit $E_{1} \rightarrow E(1)$ in presence of two interior fixed points.

Fig.2 This figure shows the existence of the heteroclinic orbit $E_{1} \rightarrow E_{2}$ above the prey isocline in presence of two interior fixed points.

Fig. 3 This figure shows the existence of a 2-disc of connections $E(1) \rightarrow E_{0}$. The boundary of the disc contains the connections $E_{1} \rightarrow E_{0}$ and $E(2) \rightarrow E_{0}$. 
Figures

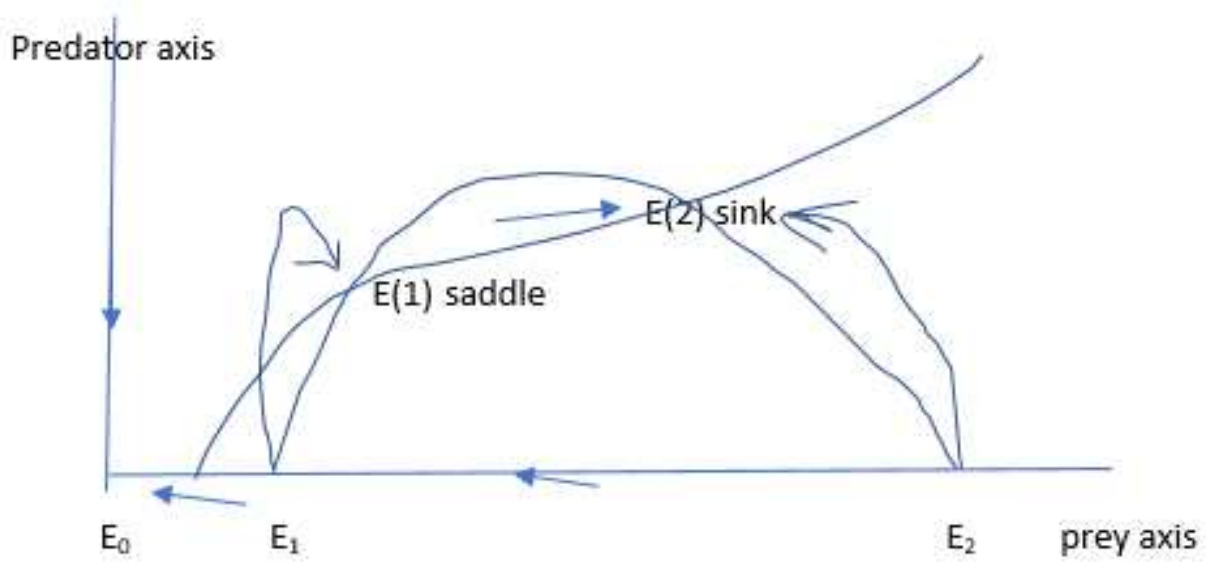

Figure 1

This figure shows the existence of the heteroclinic orbit $E 1 \otimes E(1)$ in presence of two interior fixed points.

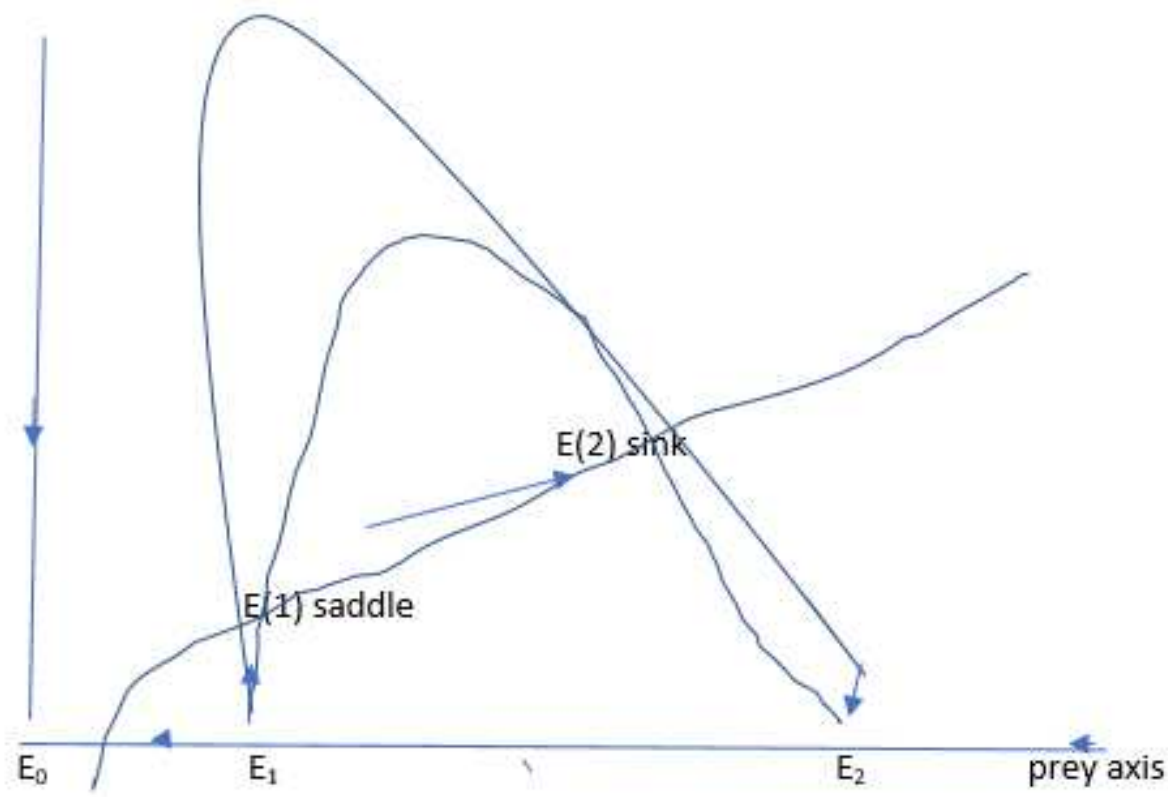

Figure 2

This figure shows the existence of the heteroclinic orbit E1 $₫ \mathrm{E} 2$ above the prey isocline in presence of two interior fixed points. 


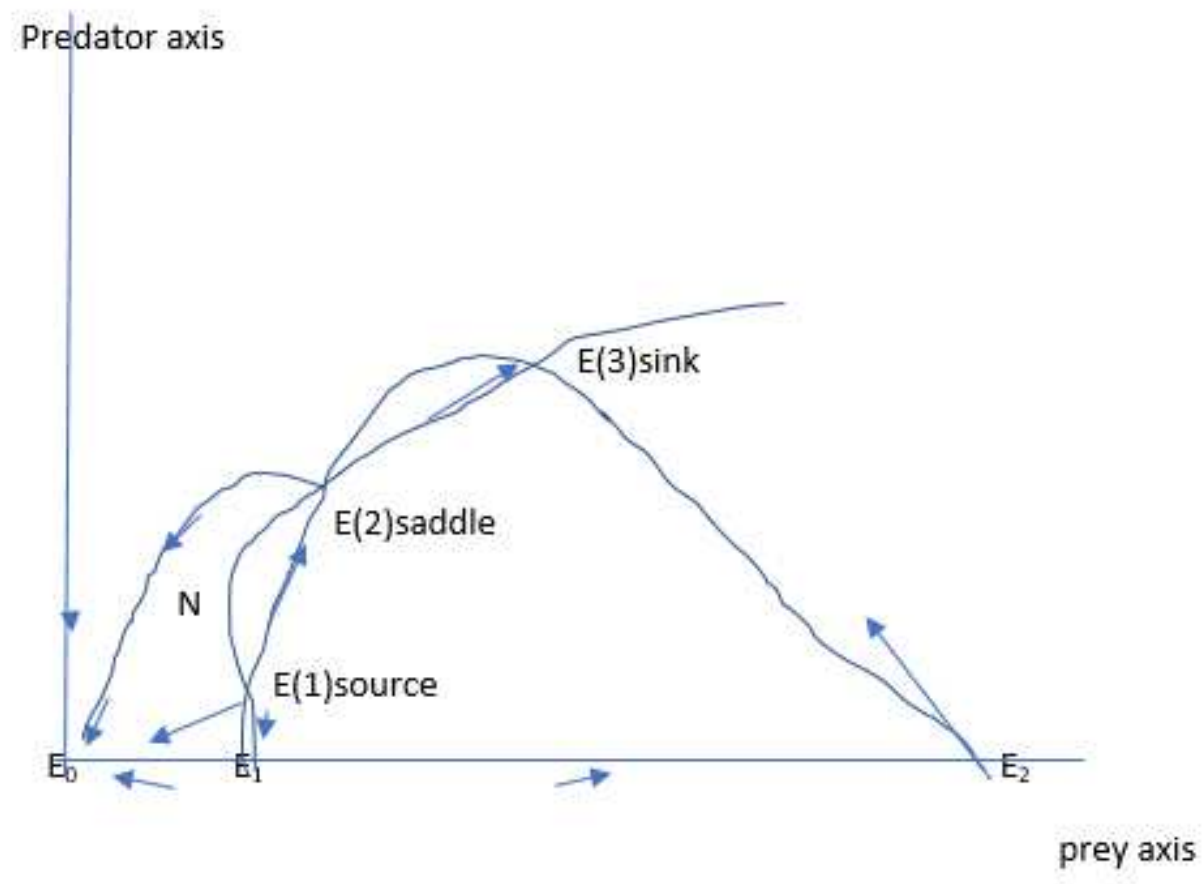

Figure 3

This figure shows the existence of a 2-disc of connections $E(1) \otimes E 0$. The boundary of the disc contains the connections $\mathrm{E} 1 \otimes \mathrm{E} 0$ and $\mathrm{E}(2) \otimes \mathrm{E} 0$. 